Научная статья

УДК 532.2

DOI: 10.18101/2304-5728-2021-4-26-33

\title{
УРАВНЕНИЕ ОРНШТЕЙНА - ЦЕРНИКЕ В ФИЗИКЕ СТЕКЛООБРАЗНОГО СОСТОЯНИЯ
}

\section{(с) Цыдыпов Шулун Балдоржиевич}

доктор технических наук, доцент, Бурятский государственный университет имени Доржи Банзарова Россия, 670000, г. Улан-Удэ, ул. Смолина, 24а shulun52@gmail.com

\section{(C) Герман Евгений Иванович}

старший преподаватель,

Бурятский государственный университет имени Доржи Банзарова Россия, 670000, г. Улан-Удэ, ул. Смолина, 24а net-admin@list.ru

\section{(C) Аграфонов Юрий Васильевич}

доктор физико-математических наук, профессор,

Иркутский государственный университет

Россия, 664003, г. Иркутск, ул. К. Маркса, 1

agrafonov@physdep.isu.ru

Аннотация. Показано, что уравнение Орнштейна - Цернике физики классических жидкостей для равновесных состояний пространственно-однородных изотропных систем применимо для расчета теплофизических характеристик систем частиц с центральными силами межчастичного взаимодействия в неравновесном стеклообразном состоянии. Для использования уравнения Орнштейна - Цернике в описании микроскопически неоднородных стеклообразных тел в уравнение введена одночастичная функция распределения, учитывающая различия между структурами жидкости и аморфного твердого тела. Расчет теплоемкости и скорости звука в аргоне показывает удовлетворительное согласие с результатами численного и натурного эксперимента, что свидетельствуют о возможности описания систем частиц в стеклообразном состоянии с помощью модифицированного уравнения Орнштейна - Цернике. При численном решении этого уравнения в физике классических жидкостей наиболее часто используется быстросходящийся алгоритм Лабика - Малиевского, который и использован в данной работе.

Ключевые слова: стеклообразное состояние, частичные функции распределения, фазовый переход, уравнение Орнштейна - Цернике, численное решение интегральных уравнений.

\section{Для цитирования}

Цылыппов Ш. Б., Герман Е. И., Аграфонов Ю. В. Уравнение Орнштейна Цернике в физике стеклообразного состояния // Вестник Бурятского государственного университета. Математика, информатика. 2021. № 4. С. 26-33. 
Ш. Б. Цыљыпов, Е. И. Герман, Ю. В. Аграфонов. Уравнение Орнштейна - Цернике в физике стеклообразного состояния

\section{Введение}

Под стеклом обычно понимают любой материал неорганического или органического происхождения, у которого отсутствует дальний порядок в структуре и вязкость которого выше $10^{13}-10^{14}$ Пуаз. Согласно молекулярно-кинетическим представлениям [1-3], в принципе все вещества могут быть получены в стеклообразном состоянии, если этому переходу не помешает кристаллизация. Можно осуществить переохлаждение и переход в стекло даже расплавов металлов и получить так называемые металлические стекла [3]. Поэтому, стеклообразное состояние часто определяют как неравновесное метастабильное состояние жидкости с замороженной структурой $[4 ; 5]$.

Теплофизические и другие свойства стеклообразных твердых тел во многом определяются структурой жидкости, которая фиксируется в области стеклования. Вместе с тем в настоящее время нет общепризнанной теории перехода жидкость - стекло, а также нет полной ясности в природе жидкого и стеклообразного состояний вещества. Построение строгой статистической теории встречает большие трудности. Успехи, достигнутые в последнее время, касаются лишь равновесных свойств простых жидкостей со сферически симметричным парным потенциалом взаимодействия.

При исследовании методами статистической физики однородных и изотропных жидкостей в равновесном состоянии классикой стало использование уравнение Орнштейна - Цернике (ОЦ) [6-8]. Как показано в $[7 ; 8]$, это уравнение является прямым следствием распределения Гиббса, если учитывать только парные взаимодействия частиц. В системе частиц с вышеуказанными свойствами уравнение ОЦ отражает пространственные корреляции между положениями любых трех частиц во всем объеме системы:

$$
h\left(r_{12}\right)=C\left(r_{12}\right)+n \int C\left(r_{13}\right) h\left(r_{32}\right) d V_{3} .
$$

Здесь $r_{12}=\left|\vec{r}_{1}-\vec{r}_{2}\right|, \quad r_{13}=\left|\vec{r}_{1}-\vec{r}_{3}\right|, \quad r_{32}=\left|\vec{r}_{3}-\vec{r}_{2}\right|$ - модули приращений радиус-векторов трех произвольно взятых частиц системы, коррелирующих между собой; $h\left(r_{12}\right)$ - полная и $C\left(r_{12}\right)$ - прямая корреляционные функции; $n$ - концентрация частиц; интегрирование ведется по координатам третьей частицы по всему объему системы.

\section{1 Применение уравнения Орнштейна - Цернике к стеклообразному состоянию}

Использование уравнения ОЦ к описанию метастабильных состояний, к которым можно отнести стеклообразное состояние, является проблематичным [7; 8]. Однако, стеклообразное состояние в отличие от метастабильных жидкостей характеризуется большими временами релаксации частиц системы к структуре равновесных состояний, что позволяет применять уравнение ОЦ к стеклам, как к жидкостям с практически замороженной структурой. 
В работе Малиевского с соавторами [9] было показано, что расщепление второго максимума радиальной функции распределения на два пика в системе твердых сфер, полученные с помощью уравнения ОЦ при плотностях твердого состояния, можно считать признаком аморфного состояния. Это предположение было подтверждено экспериментами по нейтронному и рентгеновскому рассеянию, и расщепление второго максимума корреляционной функции считается признаком аморфного состояния $[10 ; 11]$. Кроме этого, моделирование методом молекулярной динамики аморфного состояния в системе Леннарда - Джонса [12] также показывают такое расщепление.

Функцию $h(r)$ в уравнении Орнштейна - Цернике (1) можно выразить через потенциал взаимодействия $\varphi(r)$ и через бридж-функционал $B[h(r)]:$

$$
h(r)=e^{-\frac{\varphi(r)}{k T}+\gamma(r)+B[h(r)]}-1,
$$

где $k$ - постоянная Больцмана, $\gamma(r)$ - непрямая корреляционная функция, равная разности между полной $h(r)$ и прямой $C(r)$ - корреляционными функциями: $h(r)-C(r)$.

Ввиду невозможности использования в (2) бридж-функционала $B[h(r)]$ в виде бесконечного ряда диаграмм, при решении уравнения ОЦ используются процедуры замыкания, устанавливающие связь между полной и прямой корреляционными функциями. В нашем случае используется замыкание Мартынова - Саркисова [8], как наиболее подходящее для описания стеклообразного состояния:

$$
B[h(r)] \rightarrow B(h(r))=-1-\gamma(r)+\frac{n F_{2}(r)}{k T}+\sqrt{1-2\left(\gamma(r)-\frac{n F_{2}(r)}{k T}\right)},
$$

где $F_{2}(r)$ - часть потенциала парного взаимодействия частиц $\varphi(r)$, отвечающая за силы притяжения между ними. В наших расчетах используется известный потенциал Леннарда - Джонса:

$$
\phi(r)=4 \varepsilon\left(\left(\frac{\sigma}{r}\right)^{12}-\left(\frac{\sigma}{r}\right)^{6}\right) .
$$

Здесь $\varepsilon-$ минимум потенциала, $\sigma$ - эффективный диаметр частиц.

Численное решение уравнения Орнштейна - Цернике (1) с использованием вышеуказанных выражений [13-16], позволяет найти двухчастичную или радиальную функцию распределения частиц $g_{2}\left(r_{12}\right)=1+h\left(r_{12}\right)$. Эта функция для однородных и изотропных систем зависит только от модуля разности радиус-векторов двух частиц $r_{12}=\left|\vec{r}_{1}-\vec{r}_{2}\right|$. Она определяет расстояние между двумя частицами в радиальном направлении. Функция радиального распределения $g_{2}\left(r_{12}\right)$ для однородной и изотропной жидкости, приведена на кривой 1 рисунка 1 . Она отражает ближний порядок частиц в жидкостях - высокий и узкий первый пик, отражающий взаимное упорядоченное расположение частиц первой координационной сферы 
Ш. Б. Цыљыпов, Е. И. Герман, Ю. В. Аграфонов. Уравнение Орнштейна - Цернике в физике стеклообразного состояния

диаметром $\sigma \cong 1$, затем быстрое убывание и расплывание по ширине следующих пиков, свидетельствующие о быстром исчезновении ближнего порядка.

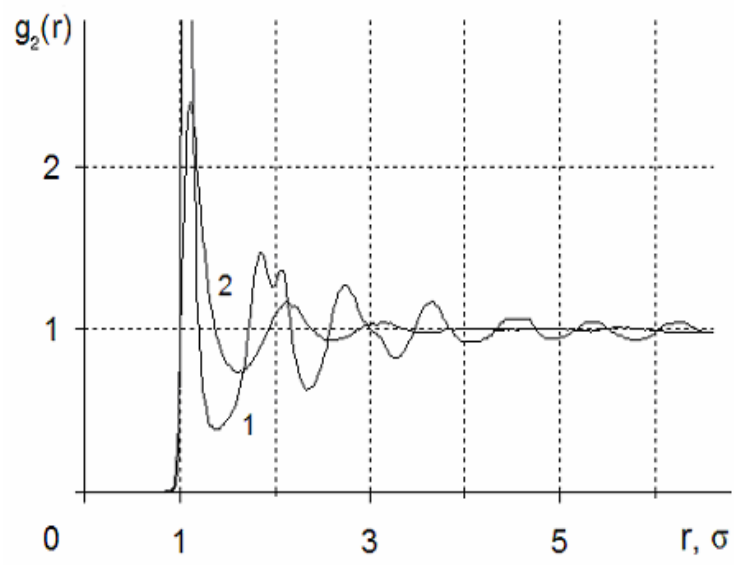

Рис. 1. Радиальная функция распределения $g_{2}(\mathrm{r})$ ЛД-системы.

1 - аморфное твердое тело, $T^{*}=0.25, n^{*}=1.1$;

2 - жидкость $T^{*}=1.0, n^{*}=0.7 ; n^{*}=n \sigma^{3}, T^{*}=T / \varepsilon$;

получено методом молекулярной динамики.

Уменьшение температуры жидкости с достаточной скоростью вблизи точки фазового перехода жидкость - кристалл приводит к тому, что при резком уменьшении межчастичного расстояния, частицы жидкости не успевают перестроиться в упорядоченную кристаллическую структуру и образуют неравновесное стеклообразное твердое тела с замороженной структурой ближнего порядка жидкости. Такой переход не относится к фазовым переходам и является кинетическим явлением с большим временем релаксации, зависящим от скорости охлаждения, от сложности структуры и сил межчастичного взаимодействия, определяющих вязкость и другие структурно чувствительные свойства системы частиц.

Переход в стеклообразное состояние отражается на виде радиальной функции распределения в виде расщепления второго максимума на два пика на кривой 1 рисунка 1 [7; 17].

Плотность стеклообразного состояния выше плотности жидкого расплава, что приводит к уменьшению простора для передвижения частиц и неоднородности, носящей микроскопический характер. Поэтому, для применения уравнения Орнштейна - Цернике (1) к стеклообразному состоянию, предлагается ввести в это уравнение функцию распределения $J\left(r_{3}\right)$, учитывающую микроскопическую неоднородность системы и зависящей только от расстояния между частицами:

$$
h\left(r_{12}\right)=C\left(r_{12}\right)+n \int J\left(r_{3}\right) C\left(r_{13}\right) h\left(r_{32}\right) d V_{3} .
$$


В стеклообразном состоянии подвижность частиц системы ограничена ближним окружением в пределах сферы с эффективным диаметром $\sigma$ :

$$
J(r)=\left\{\begin{array}{l}
1, r \leq \sigma \\
0, r>\sigma .
\end{array}\right.
$$

Подставляя (6) в выражение (5), получим замкнутое уравнения Орнштейна - Цернике пригодное для стеклообразного состояния:

$$
h\left(r_{12}\right)=C\left(r_{12}\right)+n \int\left\{\begin{array}{l}
1, r_{13} \leq \sigma \\
0, r_{13}>\sigma
\end{array}\right\} C\left(r_{13}\right) h\left(r_{32}\right) d V_{3} .
$$

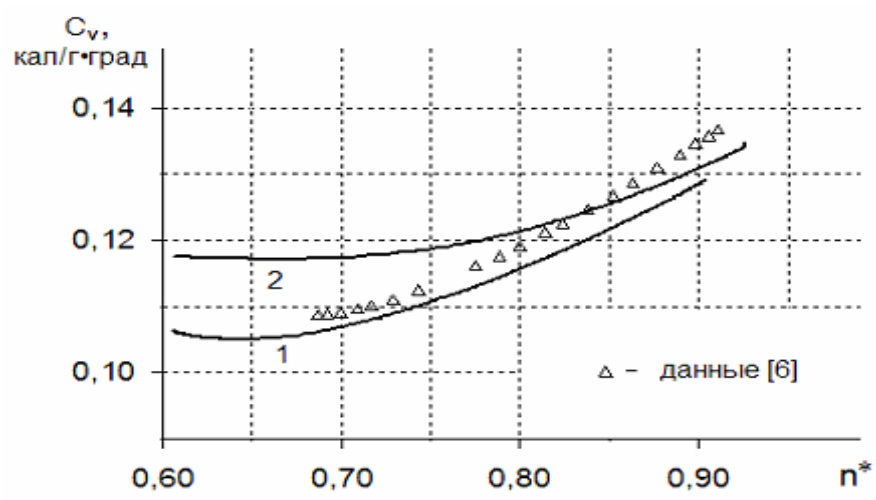

Рис. 2. Изохорическая теплоемкость аргона. $T=120 \mathrm{~K}$, 1 - уравнение (1), 2 - уравнение (7).

С использованием алгоритма Малика - Лабиевского были рассчитаны радиальные функции распределения $g_{2}\left(r_{12}\right)=1+h\left(r_{12}\right)$ для систем частиц аргона в стеклообразном состоянии при решении выражения (7).

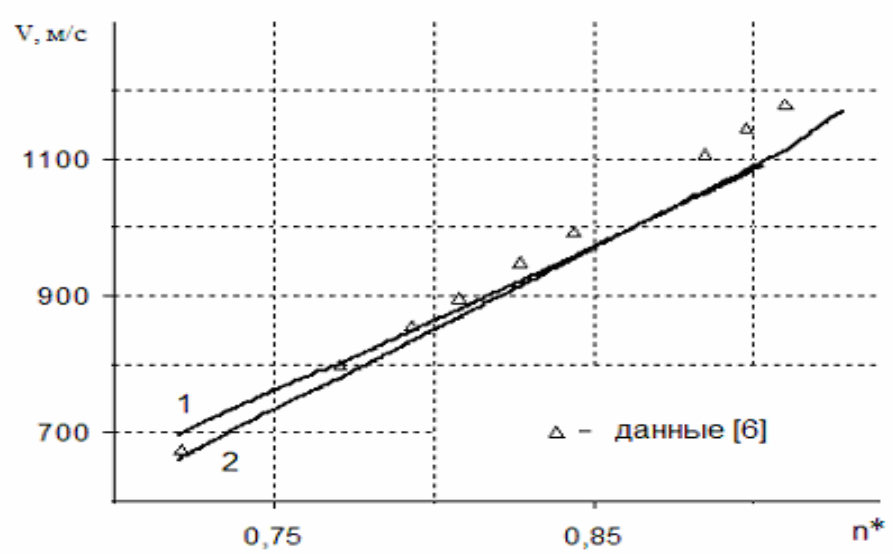

Рис. 3. Скорость звука в аргоне. $T=120 \mathrm{~K}$.

1 - уравнение (1), 2 - уравнение (7). 
Ш. Б. Цыљыпов, Е. И. Герман, Ю. В. Аграфонов. Уравнение Орнштейна - Цернике в физике стеклообразного состояния

Радиальная функция распределения позволяет рассчитать многие термодинамические свойства изучаемых систем, например теплоемкость, скорость звука и др.

На рисунках 2 и 3 приведены результаты расчетов теплоемкости и скорости звука в аргоне, показывающие, что модифицированное уравнение Орнштейна - Цернике (7) более пригодно при высоких плотностях.

Вышеприведенные результаты показывают, что модификация уравнения Орнштейна - Цернике (1) путем ввода в него одночастичной функции распределения $J\left(r_{3}\right)$, учитывающей различия между структурами жидкости и стеклообразного твердого тела позволяет получить более точные значения термодинамических параметров простых систем в микроскопически неоднородных состояниях.

Другой подход модификации уравнения Орнштейна - Цернике для исследования стеклообразного состояния на основе модели хаотического фазового перехода рассматривается в работе [18]. Для этого в уравнении Орнштейна - Цернике учитываются уравнения для прямой корреляционной функции $C\left(r_{12}\right)$, позволяющие отличить жидкое состояние от стеклообразного.

\section{Заключение}

Отличие стеклообразного состояния от жидкого должно быть каким-то образом отражено в уравнении Орнштейна - Цернике. Одним из способов учесть микроскопическую неоднородность стеклообразных тел является модификация уравнения Орнштейна - Цернике путем ввода в уравнение одночастичной функции распределения, учитывающей различия между структурами жидкости и аморфного твердого тела.

Другой способ позволяющий отличить переохлажденную жидкость от стекла заключается в модификации метода частичных функций распределения, в частности, модификации выражения для прямой корреляционной функции $C\left(r_{12}\right)$ в уравнении Орнштейна — Цернике.

Полученные интегральные уравнения физики для стеклообразных состояний являются приближенными и свидетельствуют о возможности описания аморфных состояний простых молекулярных систем с помощью модифицированного тем или иным способом уравнения Орнштейна Цернике.

\section{Литература}

1. Сандитов Д. С., Бартенев Г. М. Физические свойства неупорядоченных структур. Новосибирск: Наука, 1982. 259 с. Текст: непосредственный.

2. Mackenzie J. D. In: Modern Aspects of the Vitreous State. London, 1960. P. 1-9.

3. Egami T., Vitek V. Local Structural Fluctuations and Defect in Metallic Glasses // J. Non-Cryst. Solids. 1984. № 4(62). P. 499-510.

4. Cohen M. H., Turnbull D. Molekular Transport in Liquids and Glasses // J. Chem. Phus. 1959. № 5(31). P. 1164-1169. 
5. Turnbull D., Cohen M. H. Crystallization Kinetics and Glass Formation // Modern Aspects of the Vitreous State. London, 1960. P. 38-62.

6. Ornstein L. S., Zernike F. Contributions to the Kinetic Theory of Solids. The Thermal Pressure of Isotropic Solids // Proc. Acad. Sci. Amsterdam. 1914. Vol. 17. P. $793-$ 803.

7. Саркисов Г. Н. Молекулярные функции распределения стабильных, метастабильных и аморфных классических моделей // Успехи физических наук. 2002. № 6(172). С. 647-669. Текст: непосредственный.

8. Саркисов Г. Н. Приближённые уравнения теории жидкостей в статистической термодинамике классических жидких систем // Успехи физических наук. 1999. № 6(169). С. 625-642. Текст: непосредственный.

9. Malijevsky A., Labik S. Glass Transition and Effective Potential in the Hypernetted Chain Approximation // Smith WR Mol. Phys. 1991. Vol. 72. P. 193-200.

10. Cheng Y-T., Johnson W.L. Molecular Dynamics Computer Simulations // Science. 1987. Vol. 235. P. 997-1012.

11. Cargill G. S. Exact Derivatives of the Pair-Correlation Function of Simple Liquids Using the Tangent Linear Method // Solid State Phys. 1975. Vol. 30. P. 227-320.

12. Rahman A., Mandell M. J., McTague J. P. Molecular-dynamics Study of an Amorphous Lennard-Jones System at Low-temperature // J. Chem. Phys. 1976. Vol. 64. P. $1564-1568$.

13. Homeier H. H. H., Rast S., Krienke H. Iterative Solution of the Ornstein-Zernike Equation with Various Closures Using Vector Extrapolation // Comp. Phys. Comm. 1995. Vol. 92, № 2-3. P. 188-202.

14. Malijevsky A., Labik S. New Method for the Calculation of the Pair Correlation Function // Mol. Phys. 1987. Vol. 60. P. 663-672.

15. Parisi G., Zamponi F. The Ideal Glass Transition of Hard Spheres // The Journal of Chemical Physics. 2005. Vol. 123, № $14 . \quad$ P. 144501. https://doi.org/10.1063/1.2041507

16. Rogers F. J., Young D. A. New, Thermodynamically Consistent, Integral Equation for Simple Fluids // Physical Review A. 1984. Vol. 30, № 2. P. 999. https://doi.org/10.1103/PhysRevA.30.999

17. German E. I., Tsydypov S. B., Damdinov B. B. Calculation of Argon Compressibility at Different Cooling Rates // High Temperature. 2019. № 1(57). C. 27-31.

18. Аграфонов Ю. В., Петрушин И. С., Орлов С. С., Цыдыпов Ш. Б., Герман Е. И. Интегральное уравнение Фредгольма второго рода в статистической физике жидкостей // Вестник Бурятского государственного университета. Математика, информатика. 2020. № 3. С. 32-41. Текст: непосредственный.

Статья поступила в редакцию 06.12.2021; одобрена после рецензирования 09.12.2021; принята к публикащчи 14.12.2021. 
Ш. Б. Цыљыпов, Е. И. Герман, Ю. В. Аграфонов. Уравнение Орнштейна - Цернике в физике стеклообразного состояния

\title{
ORNSTEIN - ZERNIKE EQUATION IN THE PHYSICS \\ OF THE GLASS STATE
}

Shulun B. Tsydypov

Dr. Sci. (Technical Sciences), Associate Professor,

Banzarov Buryat State University

24a, Smolina str., Ulan-Ude, 670000, Russia

shulun52@gmail.com

Evgeniy I. German

Senior lecturer

Banzarov Buryat State University

24a, Smolina str., Ulan-Ude, 670000, Russia

net-admin@list.ru

Yury V. Agrafonov

Dr. Sci. (Phys. and Math.), Professor

Irkutsk State University,

1, Karl Marx str., Irkutsk, 664003, Russia

agrafonov@physdep.isu.ru

\begin{abstract}
It is shown that the Ornstein-Zernike equation of classical fluid physics for equilibrium states of spatially homogeneous isotropic systems can be used to calculate the thermophysical characteristics of simple molecular systems with central forces of interparticle interaction in a non-equilibrium glassy state. To use the Ornstein-Zernike equation in the description of microscopically inhomogeneous glassy bodies, a single-particle distribution function is introduced into the equation. The calculation of the heat capacity and the speed of sound in argon show satisfactory agreement with the results of numerical and full-scale experiments, which indicate the possibility of describing the glassy states of simple molecular systems using the modified Ornstein-Zernike equation. For the numerical equation's solution, the Labik-Malievsky algorithm is used.

Keywords: glassy state, partial distribution functions, phase transition, OrnsteinZernike equation, numerical solution of integral equations.

\section{For citation}

Tsydypov Sh. B., German E. I., Agrafonov Yu. V. Ornstein - Zernike Equation in the Physics of the Glass State // Bulletin of Buryat State University. Mathematics, Informatics. 2021. N. 4. Pp. 26-33.
\end{abstract}

The article was submitted 06.12.2021; approved after reviewing 09.12.2021; accepted for publication 14.12.2021. 